\title{
Electroexcitation of the Roper resonance in relativistic quark models
}

\author{
I.G. Aznauryan ${ }^{1,2}$ \\ 1 Thomas Jefferson National Accelerator Facility, Newport News, Virginia 23606, USA \\ ${ }^{2}$ Yerevan Physics Institute, 375036 Yerevan, Armenia
}

\begin{abstract}
The amplitudes of the transition $\gamma^{*} N \rightarrow P_{11}(1440)$ are calculated within light-front relativistic quark model assuming that the $P_{11}(1440)$ is the first radial excitation of the $3 q$ ground state. The results are presented along with the predictions obtained in other relativistic quark models. In comparison with the previous calculations, we have extended the range of $Q^{2}$ up to $4.5 \mathrm{GeV}^{2}$ to cover the kinematic interval of the forthcoming experimental data. Using approach based on PCAC, we have checked the relative sign between quark model predictions for the $N$ and $P_{11}(1440)$ contributions to the $\pi$ electroproduction found in previous investigations.
\end{abstract}

PACS numbers: 12.39.Ki, 13.40.Gp, 13.40.Hq, 14.20.Gk

\section{INTRODUCTION}

The amplitudes of the electroexcitation of the Roper resonance on proton are expected to be obtained from the CLAS $\pi^{+}$electroproduction data at $1.7<Q^{2}<$ $4.2 \mathrm{GeV}^{2}$. There are already results [1] extracted from the preliminary data [2, 3], and the final results will be available soon. The results at $1.7<Q^{2}<$ $4.2 \mathrm{GeV}^{2}$ combined with the previous CLAS data at $Q^{2}=0.4,0.65 \mathrm{GeV}^{2}[4,5,6]$ and with the information at $Q^{2}=0$ [7] will give us knowledge of the $Q^{2}$ evolution of the $P_{11}(1440)$ electroexcitation in wide $Q^{2}$ region. This information can be very important for understanding of the nature of the Roper resonance which has been the subject of discussions since its discovery [8], because the simplest and most natural assumption that this is a first radial excitation of the $3 q$ ground state led to the difficulties in the description of the mass of the resonance.

To use the information on the the electroexcitation of the Roper resonance, it is important to understand the quark model predictions for the $Q^{2}$ evolution of the transition $\gamma^{*} N \rightarrow P_{11}(1440)$. It is known that with increasing $Q^{2}$, when the momentum transfer becomes larger than the masses of the constituent quarks, a relativistic treatment of the electromagnetic excitations, which is important already at $Q^{2}=0$, becomes crucial. The consistent way to realize the relativistic treatment of the $\gamma^{*} N \rightarrow N^{*}$ transitions is to consider them in the lightfront (LF) dynamics [9, 10, 11]. Within this framework, one can set up an impulse approximation and avoid difficulties caused by different momenta of initial and final hadrons in the $\gamma^{*} N \rightarrow N^{*}$ transition.

For the $P_{11}(1440)$, the LF calculations have been realized in Refs. 12, 13, 14, 15, 16. However, quantitatively

Notice: Authored by The Southeastern Universities Research Association, Inc. under U.S. DOE Contract No. DE-AC05-84150. The U.S. Government retains a non-exclusive, paid-up, irrevocable, world-wide license to publish or reproduce this manuscript for U.S. Government purposes. the obtained results differ from each other and there are inconsistencies in the signs of the amplitudes presented in Refs. 12, 13, 14, 15, 16. In particular, the relative sign between the $N N \pi$ and $P_{11}(1440) N \pi$ amplitudes, which is necessary for comparison of quark-model predictions for the $\gamma^{*} N \rightarrow P_{11}(1440)$ amplitudes with experimental data, was found and taken into account only in Ref. [13], where for this purpose the ${ }^{3} P_{0}$ model of Ref. [17] was used.

For this reason, we found it important to perform calculations of the $\gamma^{*} N \rightarrow P_{11}(1440)$ amplitudes in the LF relativistic quark model, and also to check the relative sign between the $N N \pi$ and $P_{11}(1440) N \pi$ amplitudes, using a different approach. We will present and discuss the results of all relativistic quark models. All results will be given within a unified definition of the $\gamma^{*} N \rightarrow P_{11}$ (1440) helicity amplitudes.

We will find the relative sign between the $N N \pi$ and $P_{11}(1440) N \pi$ amplitudes by using the hypothesis of partially conserved axial-vector current (PCAC) in the way used in Ref. [18] for the description of the hadron $\rightarrow$ hadron $+\pi$ transitions. This method was previously used in Ref. [19] for evaluation of the relative signs between the $N N \pi$ and $N^{*} N \pi$ amplitudes for the resonances of the multiplet $\left[70,1^{-}\right]$. Using an approach based on PCAC we have confirmed the sign obtained in Ref. 13].

In Sec. II we will specify definitions of the $\gamma^{*} p \rightarrow$ $P_{11}(1440)$ helicity amplitudes in quark models and will give relations between these amplitudes and the amplitudes extracted from experimental data. In Sec. III the formulas for the $\gamma^{*} p \rightarrow P_{11}$ (1440) amplitudes obtained in the LF relativistic quark model of Ref. [20] will be given. The relative sign between the $N N \pi$ and $P_{11}(1440) N \pi$ amplitudes will be found in Sec. IV. The results of numerical calculations of the $\gamma^{*} p \rightarrow P_{11}(1440)$ amplitudes will be presented and discussed in Sec. $\mathrm{V}$ along with the results obtained in Refs. [12, 13, 14, 15, 16]. Summary will be done in Sec. VI. 


\section{DEFINITIONS OF THE $\gamma^{*} N \rightarrow P_{11}(1440)$ HELICITY AMPLITUDES}

The relations between the $\gamma^{*} p \rightarrow P_{11}(1440)$ helicity amplitudes extracted from $\pi$ electroproduction data and the $P_{11}(1440)$ contribution to the $\gamma^{*} p \rightarrow \pi N$ multipole amplitudes have the following form [21, 22]:

$$
\begin{aligned}
& A_{1 / 2}=a \operatorname{Im} M_{1-}^{R}(W=M), \\
& S_{1 / 2}=-\frac{a}{\sqrt{2}} \operatorname{Im} S_{1-}^{R}(W=M), \\
& a=\sqrt{2 \pi \frac{q_{\pi}}{K} \frac{M}{m} \frac{\Gamma}{\beta_{\pi N}}} / C, \\
& C=-\sqrt{\frac{1}{3}} \text { for } \gamma^{*} p \rightarrow p \pi^{0}, \\
& C=-\sqrt{\frac{2}{3}} \text { for } \gamma^{*} p \rightarrow n \pi^{+} .
\end{aligned}
$$

Here $\Gamma$ and $M$ are the total width and mass of the resonance, $\beta_{\pi N}$ is its branching ratio to $\pi N$ channel, $m$ is the mass of the nucleon, $K \equiv\left(M^{2}-m^{2}\right) / 2 M, q_{\pi}$ is the center of mass momentum of the pion at the resonance position.

The helicity amplitudes defined through Eqs. (1-5) include the relative sign between $g_{N N \pi}$ and $g_{R N \pi}$ coupling constants that determine the relative contributions of the Born term and the $P_{11}(1440)$ to the reaction $\gamma^{*} N \rightarrow \pi N$.

In Refs. [12, 14, 15, 16] and in this work, the quark model predictions for the transition $\gamma^{*} N \rightarrow P_{11}(1440)$ are found in terms of form factors that enter the matrix element of the transition current. Following Ref. [12], we define this matrix element in the following form:

$$
\begin{aligned}
& <N^{*}\left|J_{\mu}\right| N>=e \bar{u}\left(P^{*}\right) \Gamma_{\mu} u(P) \\
& \Gamma_{\mu}=\left(q^{2} \gamma_{\mu}-(q \gamma) q_{\mu}\right) F_{1}^{*}\left(Q^{2}\right)+i \sigma_{\mu \nu} q^{\nu} F_{2}^{*}\left(Q^{2}\right)
\end{aligned}
$$

Helicity amplitudes of the $\gamma^{*} p \rightarrow P_{11}(1440)$ transition are related to the form factors $F_{1 p}^{*}\left(Q^{2}\right)$ and $F_{2 p}^{*}\left(Q^{2}\right)$ by:

$$
\begin{gathered}
A_{1 / 2}^{q}=c\left[-Q^{2} F_{1 p}^{*}\left(Q^{2}\right)+(M+m) F_{2 p}^{*}\left(Q^{2}\right)\right], \\
S_{1 / 2}^{q}=-c \frac{q_{c m s}^{*}}{\sqrt{2}}\left[(M+m) F_{1 p}^{*}\left(Q^{2}\right)+F_{2 p}^{*}\left(Q^{2}\right)\right], \\
c=\sqrt{\pi \alpha \frac{Q^{2}+(M-m)^{2}}{M m K}}
\end{gathered}
$$

where $\alpha=e^{2} / 4 \pi=1 / 137$.

The expressions for the helicity amplitudes $A_{1 / 2}^{q}, S_{1 / 2}^{q}$ in Refs. [14, 15, 16] coincide with Eqs. (8,9). In Ref. [12], the formulas for both amplitudes contain inaccuracies in the coefficients that we have corrected in the results from that work presented below.

In quark model calculations another definition of the $\gamma^{*} N \rightarrow P_{11}(1440)$ helicity amplitudes through the $\gamma^{*} N \rightarrow N^{*}$ transition current matrix elements also is used [12, 13, 23]:

$$
\begin{aligned}
& A_{1 / 2}^{q}=b<N^{*+}, S_{z}^{*}=\frac{1}{2}|J \epsilon| p, S_{z}=-\frac{1}{2}>, \\
& S_{1 / 2}^{q}=b \frac{q_{c m s}^{*}}{Q}<N^{*+}, S_{z}^{*}=\frac{1}{2}|J \epsilon| p, S_{z}=\frac{1}{2}>, \\
& b=\left[\frac{2 \pi \alpha}{K}\right]^{1 / 2} .
\end{aligned}
$$

Here it is supposed that the virtual photon moves along the z-axis in the $N^{*}$ rest frame and its 3 -momentum is $q_{c m s}^{*}, P^{*}=P+q, Q^{2} \equiv-q^{2}$.

The helicity amplitudes $A_{1 / 2}^{q}, S_{1 / 2}^{q}$ found in different works can not be directly compared with each other and with amplitudes extracted from experimental data for the following reasons:

(i) The signs of the amplitudes $A_{1 / 2}^{q}, S_{1 / 2}^{q}$ in quark models depend on the sign of the $P_{11}(1440)$ wave function, which is different in different works. Let us define the $P_{11}(1440)$ wave function in the nonrelativistic approximation in the form:

$$
\Psi_{R} \sim \zeta\left(\sum \mathbf{k}_{i}^{2}-\alpha^{2}\right) \Psi_{N},
$$

where $\mathbf{k}_{i}(i=1,2,3)$ are the quark three-momenta in the center-of-mass system and $\alpha$ is the parameter that depends on interquark forces. With this definition, the wave functions of Refs. [12, 14, 15, 16] and Ref. 13] correspond to $\zeta=+$ and - , respectively. Our definition of the sign of the $P_{11}(1440)$ wave function used below coincides with that in Ref. [13].

(ii) The amplitudes $A_{1 / 2}^{q}, S_{1 / 2}^{q}$ found in quark models through relations (8-11) are related to the amplitudes $A_{1 / 2}, S_{1 / 2}$ extracted from experimental data using definitions (1-5) in the following way

$$
A_{1 / 2}=-\xi_{R} A_{1 / 2}^{q}, S_{1 / 2}=-\xi_{R} S_{1 / 2}^{q} .
$$

Here $\xi_{R}$ is the relative sign between $g_{N N \pi}$ and $g_{R N \pi}$ coupling constants, which also depends on $\zeta$. The sign $\zeta$ drops out from the amplitudes $A_{1 / 2}, S_{1 / 2}$, therefore, these amplitudes do not depend on the sign of the $P_{11}(1440)$ wave function.

In the previous investigations, the sign $\xi_{R}$ has been found and taken into account only in Ref. [13], where it was obtained that $\xi_{R}=-\zeta$. We have obtained the same sign in this work using a different approach.

In Refs. [12, 14, 15, 16], only the results for the amplitudes $A_{1 / 2}^{q}, S_{1 / 2}^{q}$ are given. Corresponding results for the amplitudes $A_{1 / 2}, S_{1 / 2}$ we will present using the sign $\xi_{R}$ found in this work and in Ref. [13].

\section{THE $\gamma^{*} p \rightarrow P_{11}(1440)$ AMPLITUDES IN THE RELATIVISTIC QUARK MODEL}

The calculations of the $\gamma^{*} N \rightarrow P_{11}(1440)$ amplitudes we have performed in the relativistic quark model of Ref. 
[20], constructed for radiative transitions of hadrons in the infinite momentum frame (IMF), where

$$
\begin{aligned}
& \mathbf{P} \| z, \quad P \rightarrow \infty, \\
& q=\left(\mathbf{q}_{\perp},-\frac{M^{2}-m^{2}-\mathbf{q}_{\perp}^{2}}{4 P}, \frac{M^{2}-m^{2}-\mathbf{q}_{\perp}^{2}}{4 P}\right) .
\end{aligned}
$$

Here $P$ is the momentum of the initial hadron and $q$ is the photon momentum, $Q^{2}=\mathbf{q}^{2}$. Such approach is analogous to the LF calculations $9,10,11,12,13,14$, 15, 16]. The derivation of the formulas for the transition $\gamma^{*} N\left(\frac{1}{2}^{+}\right) \rightarrow N\left(\frac{1}{2}^{+}\right)$are presented in detail in Ref. [24], where the model of Ref. [20] was used for investigation of the $Q^{2}$ evolution of the nucleon form factors. Here we will give final expressions for the form factors $F_{1 p}^{*}\left(Q^{2}\right)$, $F_{2 p}^{*}\left(Q^{2}\right)$ that follow from the results of Ref. [24]:

$$
\begin{aligned}
& Q^{2} F_{1 p}^{*}\left(Q^{2}\right)=\int \mathcal{F}_{1 p} \Phi_{N}\left(M_{0}^{2}\right) \Phi_{R}\left(M_{0}^{\prime 2}\right) d \Gamma \\
& Q F_{2 p}^{*}\left(Q^{2}\right)=\int \mathcal{F}_{2 p} \Phi_{N}\left(M_{0}^{2}\right) \Phi_{R}\left(M_{0}^{\prime 2}\right) d \Gamma
\end{aligned}
$$

where $\Phi_{N}\left(M_{0}^{2}\right)$ and $\Phi_{R}\left(M_{0}^{\prime 2}\right)$ are radial parts of the nucleon and $P_{11}(1440)$ wave functions, $M_{0}$ and $M_{0}^{\prime}$ are invariant masses of quarks in the initial and final hadrons.

Let us parametrize quark momenta in the initial and final hadrons by:

$$
\mathbf{p}_{i}=x_{i} \mathbf{P}+\mathbf{k}_{i \perp}, \quad \mathbf{p}_{i}^{\prime}=x_{i} \mathbf{P}^{\prime}+\mathbf{k}_{i \perp}^{\prime},
$$

where $i=a, b, c$ denotes the quarks in the hadrons, and we will suppose that the current interacts with the quark a. It is convenient to parametrize the variables $x_{i}, \mathbf{k}_{i \perp}$ and $\mathbf{k}_{i \perp}^{\prime}$ in the following way:

$$
\begin{aligned}
& \mathbf{k}_{i \perp}=\mathbf{K}_{i \perp}-\frac{1}{2} y_{i} Q, \quad \mathbf{k}_{i \perp}^{\prime}=\mathbf{K}_{i \perp}+\frac{1}{2} y_{i} Q \\
& x_{a}=1-\eta, \quad y_{a}=x_{a}-1, \quad \mathbf{K}_{a \perp}=-\mathbf{K}_{\perp} \\
& x_{b}=(1-\xi) \eta, y_{b}=x_{b}, \mathbf{K}_{b \perp}=-\mathbf{k}_{\perp}+(1-\xi) \mathbf{K}_{\perp}, \\
& x_{c}=\xi \eta, \quad y_{c}=x_{c}, \quad \mathbf{K}_{c \perp}=\mathbf{k}_{\perp}+\xi \mathbf{K}_{\perp} .
\end{aligned}
$$

In terms of the variables $\mathbf{k}_{\perp}, \mathbf{K}_{\perp}, \xi, \eta$ the phase space volume has the form

$$
4(2 \pi)^{6} d \Gamma=\frac{d \mathbf{k}_{\perp} d \mathbf{K}_{\perp} d \xi d \eta}{\xi(1-\xi) \eta(1-\eta)},
$$

the invariant masses of quarks are equal to

$$
\begin{aligned}
& M_{0}^{2}\left(M_{0}^{\prime 2}\right)=\frac{\mathbf{K}_{\perp}^{2}+m_{q}^{2} \eta}{\eta(1-\eta)}+\frac{\mathbf{k}_{\perp}^{2}+m_{q}^{2}}{\eta \xi(1-\xi)} \\
& +\frac{\eta Q^{2}}{4(1-\eta)} \mp \frac{K_{x} Q}{1-\eta},
\end{aligned}
$$

and the functions $\mathcal{F}_{1 p}, \mathcal{F}_{2 p}$ in Eqs. (15) and (16) have the form

$$
\begin{aligned}
& \mathcal{F}_{1 p}=\frac{R_{++}^{a}\left(R_{++}^{b} R_{--}^{c}-R_{+-}^{b} R_{-+}^{c}\right)}{D_{a} D_{b} D_{c}}, \\
& \mathcal{F}_{2 p}=\frac{R_{-+}^{a}\left(R_{-+}^{b} R_{+-}^{c}-R_{--}^{b} R_{++}^{c}\right)}{D_{a} D_{b} D_{c}},
\end{aligned}
$$

where

$$
\begin{aligned}
R_{ \pm \pm}^{i}= & m_{i} m_{i}^{\prime}+\mathbf{K}_{i \perp}^{2}-\frac{1}{4} y_{i}^{2} Q^{2} \pm i y_{i} Q K_{i y} \\
R_{ \pm \mp}^{i}= & \mp \frac{1}{2} y_{i}\left(m_{i}+m_{i}^{\prime}\right) Q \\
& +x_{i}\left(M_{0}-M_{0}^{\prime}\right)\left(i K_{i y} \mp K_{i x}\right) \\
m_{i}= & m_{q}+M_{0} x_{i}, \quad m_{i}^{\prime}=m_{q}+M_{0}^{\prime} x_{i} \\
D_{i}= & {\left[\left(m_{i}^{2}+\mathbf{k}_{i \perp}^{2}\right)\left(m_{i}^{2}+\mathbf{k}_{i \perp}^{\prime 2}\right)\right]^{1 / 2} }
\end{aligned}
$$

$m_{q}$ is the quark mass, $i=a, b, c$.

Under the assumption that $P_{11}(1440)$ is a radial excitation of the nucleon considered as the $3 q$ ground state, we have defined $\Phi_{R}\left(M_{0}^{2}\right)$ in the form:

$$
\Phi_{R}\left(M_{0}^{2}\right)=N\left(\beta^{2}-M_{0}^{2}\right) \Phi_{N}\left(M_{0}^{2}\right) .
$$

The parameters $N$ and $\beta$ are determined by the conditions:

$$
\int \Phi_{R}\left(M_{0}^{2}\right) \Phi_{N}\left(M_{0}^{2}\right) d \Gamma=0, \quad \int \Phi_{N(R)}^{2}\left(M_{0}^{2}\right) d \Gamma=1 .
$$

As in Refs. [20, 24], we will take the radial part of the nucleon wave function in the Gaussian form: $\Phi_{N}\left(M_{0}^{2}\right) \sim$ $\exp \left(-M_{0}^{2} / 6 \alpha_{H O}^{2}\right)$. The only parameters of the approach, the quark mass $\left(m_{q}=0.22 \mathrm{GeV}\right)$ and the harmonicoscillator parameter $\left(\alpha_{H O}=0.38 \mathrm{GeV}\right)$, were found in Ref. [20] from the description of the static properties of the nucleon.

\section{THE RELATIVE SIGN BETWEEN THE $N N \pi$ AND $P_{11}(1440) N \pi$ AMPLITUDES}

In order to find the relative sign between the $N N \pi$ and $P_{11}(1440) N \pi$ amplitudes, we utilize PCAC in the way used in Ref. [18] for the description of the hadron $\rightarrow$ hadron $+\pi$ transitions. PCAC relates the divergence of the charged axial-vector current to the pion field:

$$
\partial_{\lambda}\left(J_{1}^{a \lambda}+i J_{2}^{a, \lambda}\right)=m_{\pi}^{2} f_{\pi} \phi_{\pi^{-}},
$$

where $f_{\pi}=132 \mathrm{MeV}$ is the $\pi \rightarrow \mu \nu$ coupling constant.

In IMF (14), the matrix element of Eq. (29) between two spin $\frac{1}{2}$ particles with momenta $P_{1}$ and $P_{2}$ at $\mathbf{q}_{\perp}=0$ gives:

$$
f_{\pi} g\left(N_{1} N_{2} \pi\right)=g_{A}^{12} \frac{m_{1}+m_{2}}{\sqrt{2}}
$$

where

$$
g_{A}^{12}=\frac{1}{2 P}<P_{2}, S_{z}=\frac{1}{2}\left|J_{1+i 2}^{a, 0}\right| P_{1}, S_{z}=\frac{1}{2}>\left.\right|_{P \rightarrow \infty}
$$

and $g\left(N_{1} N_{2} \pi\right)$ is the coupling constant in the $N_{1} N_{2} \pi$ vertex:

$$
\mathcal{L}_{N_{1} N_{2} \pi}=i g\left(N_{1} N_{2} \pi\right) \bar{\psi}_{2} \gamma_{5} \vec{\tau} \psi_{1} \vec{\phi}
$$


According to the results of Ref. [20], we have:

$$
g_{A}^{12}=\frac{5}{3} \int \frac{\left(m_{q}+M_{0} x_{a}\right)^{2}-\mathbf{K}_{\perp}^{2}}{\left(m_{q}+M_{0} x_{a}\right)^{2}+\mathbf{K}_{\perp}^{2}} \Phi_{1}\left(M_{0}^{2}\right) \Phi_{2}\left(M_{0}^{2}\right) d \Gamma .
$$

Using Eqs. (30) and (33) one can find the relative sign between the $N N \pi$ and $P_{11}(1440) N \pi$ coupling constants: $\xi_{R}$. In the limit when $\alpha_{H O} \ll m_{q}$, the ratio of the coupling constants (31) for the $P_{11}(1440) N$ and $N N$ transitions that follows from Eq. (33) can be found in analytical form and is equal to:

$$
\frac{g_{A}^{R N}}{g_{A}^{N N}}=-\zeta \frac{\alpha_{H O}^{2}}{3 \sqrt{6} m_{q}^{2}} .
$$

We have also calculated the ratio (34) numerically for the values of the quark mass in the range $0<m_{q}<m / 3$ and in the wide range of the harmonic oscillator parameter which includes the values of $\alpha_{H O}$ from Refs. [12, 13, 20]. For all these values of $m_{q}$ and $\alpha_{H O}$, we have found the same sign as in Eq. (34). So, using approach based on PCAC we have obtained that the relative sign between $N N \pi$ and $P_{11}(1440) N \pi$ coupling constants is equal to $\xi_{R}=-\zeta$. The same sign was obtained in Ref. [13] using ${ }^{3} P_{0}$ model.

\section{RESULTS}

Our results for the $\gamma^{*} p \rightarrow P_{11}(1440)$ helicity amplitudes are presented in Fig. 1 along with the results obtained in Refs. [12, 13, 14, 15, 16] and with experimental data. The thick lines correspond to the results obtained in the LF formalism using the plus component of the electromagnetic current $J^{+} \equiv J^{0}+J^{3}$ in the frame where $q^{+} \equiv q^{0}+q^{3}=0$. These results are more favorable, because in this case the contributions of virtual $q \bar{q}$ pairs are eliminated, and the processes $\gamma^{*} N \rightarrow N^{*}$ are determined only by the $N\left(N^{*}\right) \rightarrow 3 q$ vertices (Refs. $[9,10,11,20,25])$. Therefore, the matrix elements of $J^{+}$ in the frame $q^{+}=0$ can be presented in analogy with the nonrelativistic case through a sum of one-body currents for constituent quarks. Let us stress that in such approach when we evaluate electromagnetic form factors by utilizing only plus component of the electromagnetic current, it is supposed that other components have proper behavior in order to fulfill the current conservation.

The thin dash-dotted lines are the results of Ref. 15] obtained in the LF formalism; however, in this investigation transverse component of the electromagnetic current, which can contain contributions of virtual $q \bar{q}$ pairs, is also used.

The thin solid and dashed lines correspond to the results of Ref. [16] obtained in the relativistic quark models with instant-form and point-form kinematics.

The results of all quark models presented in Fig. 1 correspond to the sign $\xi_{R}$ found in this work and in Ref. [13.

It is important to note that in all approaches 12,13 , 14, 15, 16] and in the approach used in this work, a good description of elastic nucleon form factors has been obtained. Within the approach of this work, the proton and neutron electromagnetic form factors and the nucleon axial-vector form factor were described up to $Q^{2}=4 \mathrm{GeV}^{2}$ [24]. The predictions obtained in Ref. 24] are also in reasonable agreement with the experimental data obtained later, for example, with the data from Refs. [26, 27]. A good description of nucleon form factors up to $Q^{2}=4 \mathrm{GeV}^{2}$ was obtained also in Ref. [13]. In the approaches of Refs. [14, 15, 16] the available data on nucleon form factors were described up to $Q^{2}=10 \mathrm{GeV}^{2}$. The approach of Ref. 12] describes these form factors in more narrow region of $Q^{2}: Q^{2}<1.5 \mathrm{GeV}^{2}$.

In this work and in Refs. [12, 13, 16], a simple algebraic form for the radial part of the $3 q$ ground state wave function is used. In our approach and in Refs. [12, 13], this is a Gaussian form with one parameter: the harmonicoscillator parameter. In Ref. [16], the radial part of the ground state wave function has powerlike form with two parameters. The parameters of all these approaches, except Ref. [13], were found from the description of elastic nucleon form factors in the ranges of $Q^{2}$ discussed above. In Ref. 13], the harmonic-oscillator parameter was taken from Ref. 28], were it was found from the description of baryon masses in the nonrelativistic approximation. This parameter is very close to that used in this work and found in Refs. [20, 24] from the description of the nucleon elastic form factors.

In contrast with the approaches used in this work and in Refs. [12, 13, 16], the wave functions of Refs. [14, 15] were found from the solution of the equation for the lightfront mass operator with the one-gluon exchange between quarks taken in the form used in the relativized quark model of Ref. [29]. However, it turned out that for the description of the nucleon form factors with the nucleon wave function found in this way, a significant deviation from the pointlike constituent quarks should be introduced. Let us emphasize that in Refs. [12, 13, 16, 24], the nucleon form factors were described with pointlike constituent quarks. Another difference with the wave functions used in this work and in Refs. [12, 13, 16] is the presence of the configuration mixings in the $N$ and $P_{11}(1440)$ wave functions that arise from the one-gluon exchange between quarks.

The values of the quark mass used in this work and in Refs. [13, 14, 15, 16] are close to the light-quark mass obtained from the description of baryon masses in Ref. [29].

From Fig. 1, it can be seen that although all approaches under consideration give good description of nucleon form factors, the predictions for the $\gamma^{*} p \rightarrow$ $P_{11}(1440)$ helicity amplitudes are different. As it was mentioned above, we consider as more preferable the predictions obtained in the LF approaches based on the utilization of the plus component of the electromagnetic current. These approaches are close to each other, and the differences in their predictions are caused mainly by the differences in the $\mathrm{N}$ and $P_{11}(1440)$ wave functions. In 
spite of the differences, the predictions obtained in the LF approaches have common features that are in agreement with existing experimental data:

- The sign of the transverse amplitude $A_{1 / 2}$ at $Q^{2}=$ 0 is negative.

- The sign of the longitudinal amplitude $S_{1 / 2}$ at small $Q^{2}$ is positive.

- All LF approaches predict the change of the sign of $A_{1 / 2}$ at small $Q^{2}$. The existing experimental data show a tendency that is in agreement with such sign change. The confirmation of this prediction by forthcoming experimental data will be important for understanding of the nature of the $P_{11}(1440)$ resonance.

These predictions are obtained assuming that the $N$ and the $P_{11}(1440)$ resonance are the $3 q$ ground state and the first radial excitation of the $3 q$ ground state, respectively. The admixtures of other configuration states in the $N$ and $P_{11}(1440)$ taken into account in Refs. [14, 15] do not affect these results.

\section{SUMMARY}

In this work we have calculated the $\gamma^{*} p \rightarrow P_{11}(1440)$ transition amplitudes in the LF relativistic quark model in the range of $Q^{2}: 0 \leq Q^{2} \leq 4.5 \mathrm{GeV}^{2}$.

We have also calculated the relative sign between $g_{N N \pi}$ and $g_{R N \pi}$ coupling constants which is necessary for comparison of quark-model predictions for the $\gamma^{*} p \rightarrow$ $P_{11}(1440)$ amplitudes with those extracted from experimental data. The relative sign between the $N N \pi$ and $P_{11}(1440) N \pi$ vertices was found by relating these vertices to the matrix elements of the axial-vector current using PCAC. The obtained sign is in agreement with the sign found in Ref. 13] using different approach, namely, the ${ }^{3} P_{0}$ model of Ref. [17].

The obtained $P_{11}(1440)$ electroexcitation helicity amplitudes are presented along with the results found in relativistic quark models of Refs. [12, 13, 14, 15, 16]. All results are given using the sign $\xi_{R}$ found in this work and in Ref. [13].

All approaches under consideration give good description of nucleon form factors; however the predictions for the $\gamma^{*} p \rightarrow P_{11}(1440)$ helicity amplitudes are different.
In Fig. 1, the thick lines present the results obtained in the LF approaches using the plus component of the electromagnetic current, which we consider as more favorable. These approaches are close to each other, and the differences in their predictions are caused mainly by the differences in the $N$ and $P_{11}(1440)$ wave functions. Nevertheless, there are several features which are common for the predictions of all LF approaches. One of the features is the nontrivial behavior of the transverse amplitude $A_{1 / 2}$ connected with the change of the sign of $A_{1 / 2}$ with increasing $Q^{2}$. The existing experimental data cover a very limited region of small $Q^{2}$ up to $0.65 \mathrm{GeV}^{2}$ and have a tendency that is in agreement with such behavior. The confirmation of this prediction by forthcoming experimental data will be very important for understanding of the nature of the $P_{11}(1440)$ resonance.

All approaches fail to describe the value of the transverse amplitude $A_{1 / 2}$ at $Q^{2}=0$. This can be an indication on the large pion cloud contribution to the $\gamma^{*} N \rightarrow P_{11}(1440)$ transition that is expected to be significant at small $Q^{2}$. When the final data on the helicity amplitudes at $1.7<Q^{2}<4.2 \mathrm{GeV}^{2}$ will become available, complete simultaneous description of the nucleon form factors and the $\gamma^{*} N \rightarrow P_{11}(1440)$ amplitudes will be necessary. Such research will be important to find the size of the pion cloud contribution, to specify the form of the $N$ and $P_{11}(1440)$ wave functions, including the configuration mixings in these states, to understand the role of the quark form factors and anomalous magnetic moments, as well the role of other effects, for instance, the quark mass dependence on the quark virtualities and therefore on $Q^{2}$.

\section{ACKNOWLEDGMENTS}

I am grateful to the participants of the JLab Theory Group seminar for interest to this problem that stimulated this work, and to V.Burkert, H.Lee, A.Thomas for encouraging interest. I am thankful to B.Julia-Diaz and S.Stepanyan for their help in numerical calculations, to T.Sato for discussions of the definitions of the $\gamma^{*} N \rightarrow P_{11}(1440)$ helicity amplitudes, to H.Matevosyan for discussion of the problems related to the pion cloud contribution, and to M.Paris for valuable remarks while reading the draft of this article.
[1] I. G. Aznauryan, Proceedings of the Workshop on the Physics of Excited Nucleons, NSTAR 2005, Tallahassee, October 12-15 (World Scientific, Singapore, 2006), p.265.

[2] K. Park, Proceedings of the Workshop on the Physics of Excited Nucleons, NSTAR 2005, Tallahassee, October 12-15, (World Scientific, Singapore, 2006), p.281.

[3] K. Park, V. Burkert et al., CLAS Collaboration, to be submitted to Phys.Rev.C.

[4] I. G. Aznauryan, V. D. Burkert, H. Egiyan et al., Phys. Rev. C71, 015201 (2005).

[5] I. G. Aznauryan, V. D. Burkert et al., Phys. Rev. C72, 045201 (2005).

[6] H. Egiyan, I. G. Aznauryan, V. D. Burkert et al., Phys. Rev. C73, 025204 (2006). 

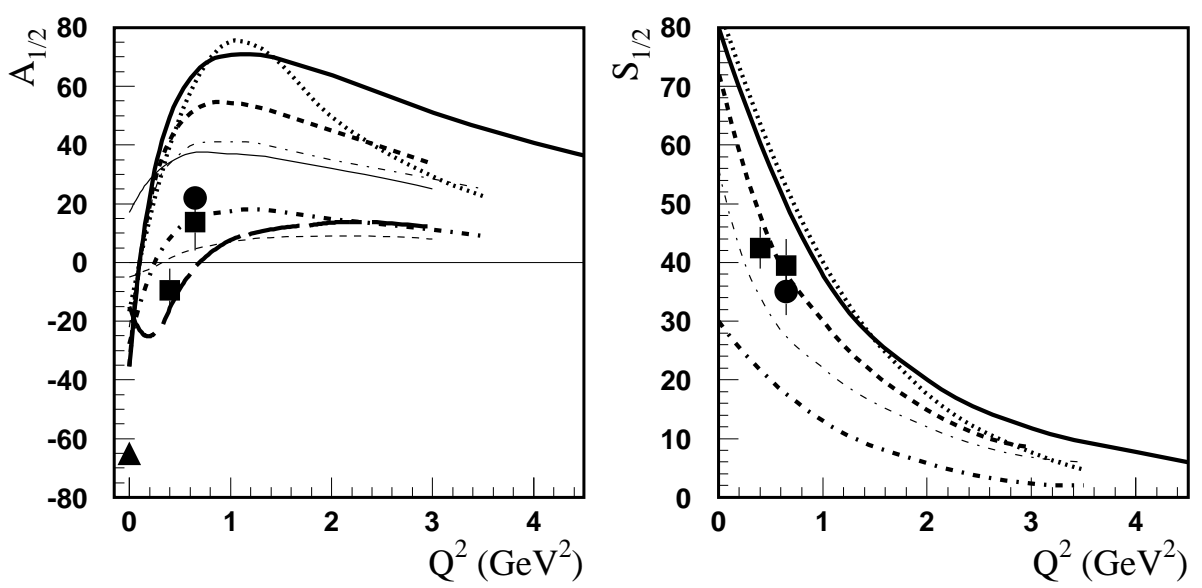

FIG. 1: Helicity amplitudes for the $\gamma^{*} p \rightarrow P_{11}(1440)$ transition (in $10^{-3} \mathrm{GeV}^{-1 / 2}$ units). Thick solid lines are the results obtained in this work. Thick dashed, dash-dotted and long-dashed lines correspond to the LF relativistic quark models of Refs. [13, 14, 16], respectively. Thick dotted lines correspond to the results of Ref. [12] corrected according to the discussion in Sec. II. Thin dash-dotted lines are the results of Ref. [15]. Thin solid and dashed lines correspond to the results of relativistic quark models with instant-form and point-form kinematics of Ref. [16]. Full boxes and circles are the results obtained in the analysis of $\pi$ electroproduction data in Ref. [4] and in the combined analysis of $\pi$ and $2 \pi$ electroproduction data in Ref. [5], respectively. Full triangle at $Q^{2}=0$ is the PDG estimate [7].

[7] Review of Particle Physics, S. Eidelman et al., Phys. Lett. B592, 1 (2004).

[8] L. Roper, Phys. Rev. Lett. 12, 340 (1964).

[9] S. D. Drell and T. M. Yan, Phys. Rev. Lett. 24, 181 (1970).

[10] V. B. Berestetskii and M. V. Terent'ev, Sov. J. Nucl. Phys., 24, 1044 (1976); 25, 347 (1977).

[11] S. J. Brodsky and S. D. Drell, Phys. Rev. D22, 2236 (1980).

[12] H. J. Weber, Phys. Rev. C41, 2783 (1990).

[13] S. Capstick and B. D. Keister, Phys. Rev. D51, 3598 (1995).

[14] F. Cardarelli, E. Pace, G. Salme, and S. Simula, Phys. Lett. B397, 13 (1997).

[15] S. Simula, Proceedings of the Workshop on the Physics of Excited Nucleons, NSTAR 2001, Mainz (Germany), March 7-10, (World Scientific, Singapore, 2001), p.135.

[16] B. Juliá-Díaz, D. O. Riska, F. Coester, Phys. Rev. C69, 035212 (2004).

[17] S. Capstick and W. Roberts, Phys. Rev. D47, 1994 (1980); Phys. Rev. D49, 4570 (1994).
[18] F. J. Gilman, M. Kugler, and S. Meshkov, Phys. Rev., D9, 715 (1974).

[19] I. G. Aznauryan and A. S. Bagdasaryan, Sov. J. Nucl. Phys. 41, 158 (1985).

[20] I. G. Aznauryan, A. S. Bagdasaryan, and N. L. TerIsaakyan, Phys. Lett. B112, 393 (1982); Yad. Fiz. 36, 1278 (1982).

[21] R. A. Arndt et al., Phys. Rev. C42, 1864 (1990).

[22] L. Tiator, D. Drechsel, S. Kamalov, and S. N. Yang, $\pi N$ Newslett. 16, 41 (2002).

[23] L. A. Copley, G. Karl, and E. Obryk, Nucl. Phys. B13, 303 (1969).

[24] I. G. Aznauryan, Phys. Lett. B316, 391 (1993); Z. für Phys. A346, 297 (1993).

[25] L. Frankfurt and M. Strikman, Nucl. Phys. B148, 107 (1979).

[26] M. K. Jones et al., Phys. Rev. Lett. 84, 1398 (2000).

[27] O. Gayou et al., Phys. Rev. Lett. 88, 092301 (2002).

[28] R. Koniuk and N. Isgur, Phys. Rev. D21, 1868 (1980).

[29] S. Capstick and N. Isgur, Phys. Rev. D34, 2809 (1986). 